This item was submitted to Loughborough's Research Repository by the author.

Items in Figshare are protected by copyright, with all rights reserved, unless otherwise indicated.

\title{
Identifying tyre models directly from vehicle test data using an extended
} Kalman filter

PLEASE CITE THE PUBLISHED VERSION

http://dx.doi.org/10.1080/00423110802684221

PUBLISHER

(C) Taylor and Francis

VERSION

AM (Accepted Manuscript)

LICENCE

CC BY-NC-ND 4.0

REPOSITORY RECORD

Best, Matt C.. 2011. "Identifying Tyre Models Directly from Vehicle Test Data Using an Extended Kalman Filter". figshare. https://hdl.handle.net/2134/8314. 
This item was submitted to Loughborough's Institutional Repository (https://dspace.lboro.ac.uk/) by the author and is made available under the following Creative Commons Licence conditions.

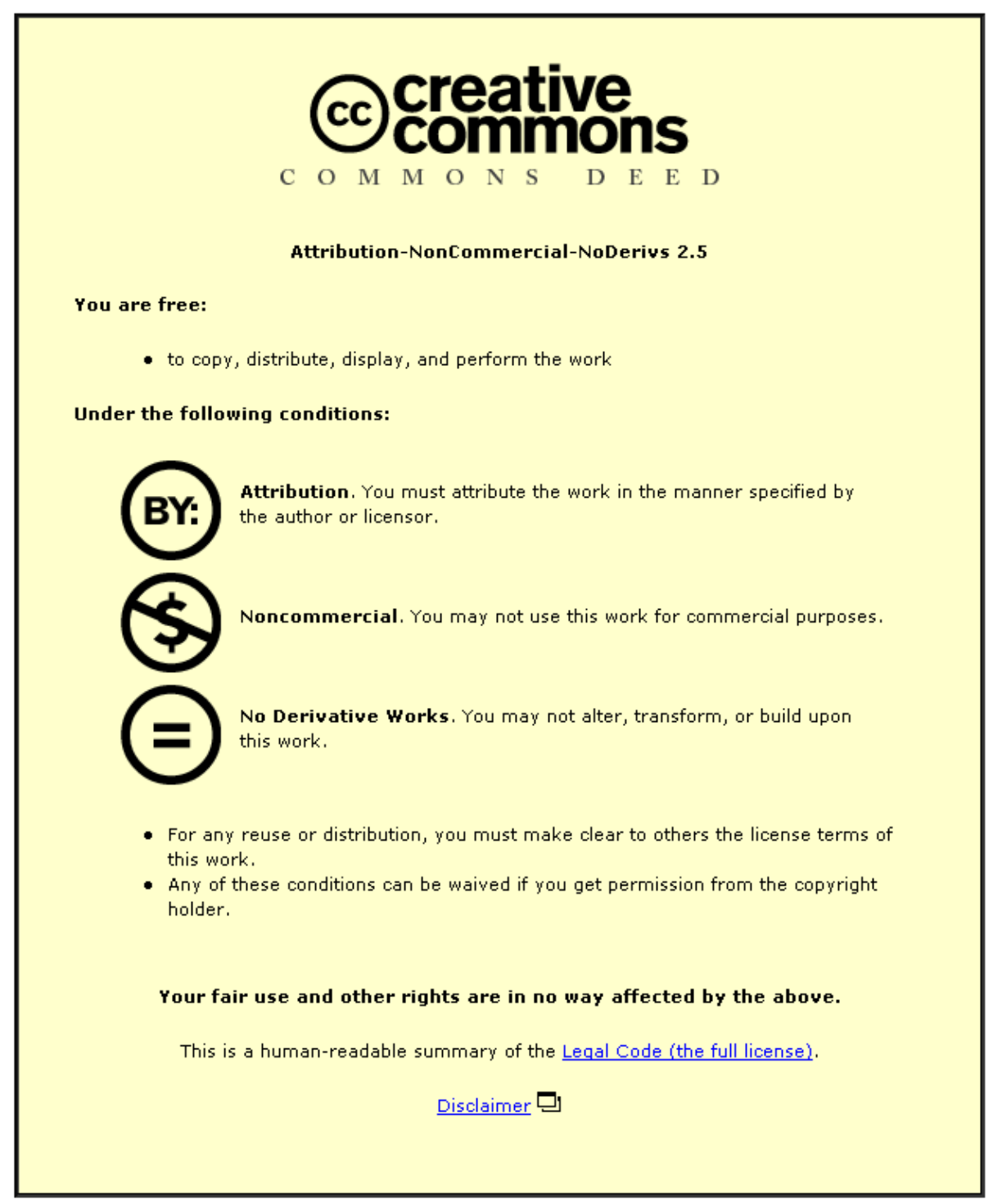

For the full text of this licence, please go to: http://creativecommons.org/licenses/by-nc-nd/2.5/ 


\title{
Identifying Tyre Models Directly from Vehicle Test Data using an Extended Kalman Filter
}

\author{
Matthew C Best \\ Dept Aeronautical and Automotive Engineering \\ Loughborough University, Leicestershire, LE11 3TU, UK \\ Phone: +44 1509227209 \\ Fax: +44 1509227275 \\ E-mail M.C.Best@lboro.ac.uk
}

\begin{abstract}
Individual tyre models are traditionally derived from component tests, with their parameters matched to force and slip measurements. They are imported into vehicle models which should, but do not always properly provide suspension geometry interaction. Recent advances in GPS / inertia vehicle instrumentation now make full state measurement viable in test vehicles, so tyre slip behaviour is directly measurable. This paper uses an Extended Kalman Filter for system identification, to derive individual load-dependent tyre models directly from these test vehicle state measurements. The resulting model therefore implicitly compensates for suspension geometry and compliance. The paper looks at two variants of the tyre model, and also considers real-time adaptation of the model to road surface friction variations. Test vehicle results are used exclusively, and the results show successful tyre model identification, improved vehicle model state prediction - particularly in lateral velocity reproduction - and an effective real-time solution for road friction estimation.
\end{abstract}

Keywords : Tyre Modelling, System Identification, Kalman Filter, Road Friction Estimation

\section{INTRODUCTION}

Sophisticated and detailed tyre models have been in development for many years, based on both rubber/steel mechanics theories and empirical results from tyre tests. The latter class, introduced by Radt and Pacejka in [1] and developed significantly since eg Pacejka [2] - have become the automatic choice in most vehicle handling models for the full range of model applications, from parametric simulation studies to control reference models, to the core of observers.

These tyre models are a very sensible option, as they have proven accuracy based on rigorously matching measured tyre responses; however the models have become complex, with well over 100 parameters in the Pacejka Delft '97 model [2], and the testing is done on the wheel and tyre in isolation from the vehicle. In applications, the whole Pacejka model is usually transferred into a vehicle model, which may itself vary in complexity from a simple bicycle framework to a full suspension component model. The value of the tyre model may therefore be diminished, since it depends on the accuracy and degree of complexity of the suspension model. Suspension compliance is a particular issue, required for the tyre model, but often not present in the vehicle model.

For a significant subset of model applications - those associated with controller and observer design in particular - it is equally, or more valuable to quantify the tyre 
properly in concert with the vehicle. The tyre model itself will inevitably be simpler, but the interactions between suspension and tyre are implicit. Thus the aim of this paper is to identify an empirical tyre model via whole vehicle test data.

Direct identification of tyre behaviour has been considered in the literature before, but most papers look only at road friction identification - eg Canudas-de-Wit et al [3]. Some models have quantified the tyre force more generally, as a function of slip, eg Bolzern et al [4] and Best et al [5], but in those papers it is the aggregated force on front / rear axle which is identified, so comparability with most single tyre models is lost. Here we seek an individual tyre model, applied equally at each of the four wheelstations, which explains the forces as a function of vertical load as well as sideslip angle. This model will clearly also absorb the suspension compliance effects, and although some compensation must be introduced to differentiate rear from front suspension configuration, it turns out this can be achieved through the addition of a single compliance parameter.

The algorithm for identification employs another familiar tool within vehicle dynamics, the Kalman filter. This is widely recognised as an effective tool for observing the dynamic states of a system, with several publications describing filters for vehicle ride and handling control. It was also the technique employed in [4] and in Best et al [6]. In those papers however, the model parameters were adapted simultaneously with propagation of a state estimator, so the available sensors drive high and low frequency correction processes simultaneously, and there are problems associated with estimating some of the matrices required in the filter design.

The technique used here is the Identifying Extended Kalman Filter (IEKF), which identifies the model alone, by the use of an estimated 'state' set comprised entirely of the parameters to be identified. The advantage of this approach is a simplification of the design parameters for the filter. The disadvantage is in the cost of the sensors which are required, since the full state set must be measurable; for the considered vehicle dynamics application, this presents the problem of measuring lateral velocity.

Recently developed GPS / inertia combination instrumentation sets are now claiming $0.1 \mathrm{~m} / \mathrm{s}$ accuracy in lateral velocity measurement however, and although these sets are expensive (costing at the time of writing around US\$30,000) they rely on combining two sensor technologies which will in future years certainly become standard in vehicles. Ironically, the signals provided by these sets are also delivered via Kalman filter algorithms, though vehicle models are not used within them.

The paper uses a 3dof yaw-roll-sideslip model as the platform for identification of the tyre model. This is the simplest platform which allows good state reproduction whilst also providing physically realistic load transfer. Although the paper does not document the process, a subset of the geometry parameters for this model is also identified using the IEKF, in advance of the tyre identification. This ensures best possible accuracy of the final model, and continues the theme of using system identification to maximise the performance available from simple model structures. This said, it would be possible to use a more complex alternative base for the identification model.

The tyre model is identified from test vehicle data, in terms of the principal Pacejka coefficients. This is done using a fixed load dependency model, and the results are compared with a version including two identified load dependence parameters. Thus the marginal performance benefit of the added degrees of freedom in the model is quantified. The IEKF is then employed with its own adaptation speed parameters altered, to establish a real-time road friction estimate to complement the batchidentified model. 


\section{THE IDENTIFYING EXTENDED KALMAN FILTER (IEKF)}

The standard Extended Kalman Filter (EKF) operates on nonlinear system and sensor models $\mathbf{f}$ and $\mathbf{h}$, which relate the true state vector $\overline{\mathbf{x}}$, measured sensor set $\mathbf{y}$, known inputs $\mathbf{u}$ and model parameters $\boldsymbol{\theta}$ at any instant $\mathrm{k}$ according to

$$
\begin{aligned}
\dot{\mathbf{x}}_{k} & =\mathbf{f}\left(\overline{\mathbf{x}}_{k}, \mathbf{u}_{k}, \boldsymbol{\theta}_{k}\right)+\boldsymbol{\omega}_{k} \\
\mathbf{y}_{k} & =\mathbf{h}\left(\overline{\mathbf{x}}_{k}, \mathbf{u}_{k}, \boldsymbol{\theta}_{k}\right)+\mathbf{v}_{k}
\end{aligned}
$$

$\boldsymbol{\omega}$ thus describes the state propagation modelling error, and $\boldsymbol{v}$ gives the sensor error. v is often misleadingly referred to as the measurement error, when in reality it aggregates measurement noise within $\mathbf{y}$, and sensor modelling errors in $\mathbf{h}$.

An optimal filter can be derived if the error sequences obey the following

$$
\begin{array}{ccc}
E\left(\boldsymbol{\omega}_{k}\right)=\mathbf{0}, & E\left(\boldsymbol{v}_{k}\right)=\mathbf{0}, & \forall k \\
E\left(\boldsymbol{\omega}_{i} \boldsymbol{\omega}_{j}^{T}\right)=\mathbf{0}, \quad E\left(\boldsymbol{v}_{i} \boldsymbol{v}_{j}^{T}\right)=\mathbf{0}, & \forall(i \neq j) \\
\mathbf{Q}_{k}=E\left(\boldsymbol{\omega}_{k} \boldsymbol{\omega}_{k}^{T}\right), \mathbf{S}_{k}=E\left(\boldsymbol{\omega}_{k} \mathbf{v}_{k}^{T}\right)=\mathbf{0}, & \mathbf{R}_{k}=E\left(\boldsymbol{v}_{k} \boldsymbol{v}_{k}^{T}\right)
\end{array}
$$

where the error covariance matrices $\mathbf{Q}_{k}, \mathbf{R}_{k}$ and $\mathbf{S}_{k}$ are assumed known. In practice these are difficult to estimate and they are often assumed to be time-invariant, and are also approximated, or even set nominally, with $\mathbf{S}$ often neglected as approximately zero.

The EKF also requires model Jacobians to be evaluated at each time step, defined

$$
\begin{aligned}
& \mathbf{F}\left(\hat{\mathbf{x}}_{k}\right)=\left.\frac{\partial \mathbf{f}\left(\mathbf{x}, \mathbf{u}_{k}, \boldsymbol{\theta}_{k}\right)}{\partial \mathbf{x}}\right|_{\mathbf{x}=\hat{\mathbf{x}}_{k}} \\
& \mathbf{H}\left(\hat{\mathbf{x}}_{k}\right)=\left.\frac{\partial \mathbf{h}\left(\mathbf{x}, \mathbf{u}_{k}, \boldsymbol{\theta}_{k}\right)}{\partial \mathbf{x}}\right|_{\mathbf{x}=\hat{\mathbf{x}}_{k}}
\end{aligned}
$$

where $\hat{\mathbf{x}}_{k}$ refers to the estimates of the state vector, and the full set of equations for the standard, real-time state estimation application are

$$
\begin{gathered}
\mathbf{F}_{k}^{*}=\mathbf{F}\left(\hat{\mathbf{x}}_{k}\right)-\mathbf{S} \mathbf{R}^{-1} \mathbf{H}\left(\hat{\mathbf{x}}_{k}\right) \\
\mathbf{K}_{k}=\mathbf{P}_{k} \mathbf{H}^{\mathbf{T}}\left(\hat{\mathbf{x}}_{k}\right)\left[\mathbf{H}\left(\hat{\mathbf{x}}_{k}\right) \mathbf{P}_{k} \mathbf{H}^{\mathbf{T}}\left(\hat{\mathbf{x}}_{k}\right)+\mathbf{R}\right]^{-1} \\
\mathbf{P}_{k}^{*}=\left[\mathbf{I}-\mathbf{K}_{k} \mathbf{H}\left(\hat{\mathbf{x}}_{k}\right)\right] \mathbf{P}_{k} \\
\mathbf{P}_{k+1}=\mathbf{P}_{k}^{*}+T\left[\mathbf{Q}-\mathbf{S} \mathbf{R}^{-1} \mathbf{S}^{\mathbf{T}}+\mathbf{F}^{*}\left(\hat{\mathbf{x}}_{k}\right) \mathbf{P}_{k}^{*}+\mathbf{P}_{k}^{*} \mathbf{F}^{* \mathbf{T}}\left(\hat{\mathbf{x}}_{k}\right)\right] \\
\hat{\mathbf{x}}_{k+1}=\hat{\mathbf{x}}_{k}+\mathbf{K}_{k}\left(\mathbf{y}_{k}-\mathbf{h}\left(\hat{\mathbf{x}}_{k}\right)\right)+T\left[\mathbf{f}\left(\hat{\mathbf{x}}_{k}\right)+\mathbf{S} \mathbf{R}^{-\mathbf{1}}\left(\mathbf{y}_{k}-\mathbf{h}\left(\hat{\mathbf{x}}_{k}\right)\right)\right]
\end{gathered}
$$

where the filter sample time $T$ is used to provide a simple Euler integration of the state derivatives.

Now the premise adopted in [4] and [6] is that an EKF can have its state vector augmented to include a subset of unknown model parameters, $\overline{\boldsymbol{\theta}}$. This vector might generally be inertia, CG geometry and/or (as in this case) tyre model parameters. The resulting filter assumes no known model for the parameter variation, and simply ensures 
slow adaptation by adjusting the expectation of errors related to the parameter changes; so eqn (1) becomes

$$
\dot{\overline{\mathbf{z}}}_{k}=\left[\begin{array}{c}
\dot{\overline{\mathbf{x}}}_{k} \\
\dot{\overline{\boldsymbol{\theta}}}
\end{array}\right]=\left[\begin{array}{c}
\mathbf{f}\left(\mathbf{x}_{k}, \mathbf{u}_{k}, \boldsymbol{\theta}_{k}\right) \\
\mathbf{0}
\end{array}\right]+\left[\begin{array}{c}
\boldsymbol{\omega}_{k}^{(\mathbf{x})} \\
\boldsymbol{\omega}_{k}^{(\boldsymbol{\theta})}
\end{array}\right]
$$

and the covariance $E\left(\boldsymbol{\omega}_{k}^{(\theta)} \boldsymbol{\omega}_{k}^{(\boldsymbol{\theta}) T}\right)$ is then set as a tuning parameter, to adjust the rate of adaptation, ensuring this is 'slow' compared to the state propagation dynamics. This method of combining state and parameter identification is attractive, and has been shown to be effective, but it relies on and is constrained by suitable estimation of the remaining error covariances.

The new identifying Kalman filter IEKF was first introduced using a simulation study in Best [7] and then validated using vehicle test data in Best et al [5]. It takes the parameter role one step further, for circumstances where all of the state variables are measurable. Provided $\mathbf{x}_{k} \in \mathbf{y}_{k}$ the state vector can be formed entirely as the set of parameters, such that eqns (1) and (2) become

$$
\begin{gathered}
\dot{\overline{\boldsymbol{\theta}}}_{k}=\boldsymbol{\omega}_{k} \\
\mathbf{y}_{k}=\mathbf{h}\left(\mathbf{y}_{k-1}, \mathbf{u}_{k-1}, \boldsymbol{\theta}_{k-1}\right)+\mathbf{v}_{k}
\end{gathered}
$$

The sensor equation is simply modified to include an Euler integrated propagation of each variable over a time step, to avoid identity equations. This reduces the system such that the entire model is represented within $\mathbf{h}$ alone. More importantly, it also reduces the system to a form where the error covariance matrices can be estimated directly from the noise sequences $\boldsymbol{\omega}_{k}$ and $\boldsymbol{v}_{k}$, which are now directly calculable.

Applying eqns (13) and (14) to the EKF formulae of eqns (7) - (11), and noting that now $\mathbf{f}=\mathbf{0}$ and $\mathbf{F}=\mathbf{0}$, we have

$$
\begin{gathered}
\mathbf{H}\left(\hat{\boldsymbol{\theta}}_{k}\right)=\left.\frac{\partial \mathbf{h}\left(\mathbf{x}_{k}, \mathbf{u}_{k}, \boldsymbol{\theta}\right)}{\partial \boldsymbol{\theta}}\right|_{\boldsymbol{\theta}=\hat{\boldsymbol{\theta}}_{k}} \\
\mathbf{K}_{k}=\mathbf{P}_{k} \mathbf{H}^{\mathbf{T}}\left(\hat{\boldsymbol{\theta}}_{k}\right)\left[\mathbf{H}\left(\hat{\boldsymbol{\theta}}_{k}\right) \mathbf{P}_{k} \mathbf{H}^{\mathbf{T}}\left(\hat{\boldsymbol{\theta}}_{k}\right)+\mathbf{R}_{k}\right]^{-1} \\
\mathbf{P}_{k}^{*}=\left[\mathbf{I}-\mathbf{K}_{k} \mathbf{H}\left(\hat{\boldsymbol{\theta}}_{k}\right)\right] \mathbf{P}_{k} \\
\mathbf{P}_{k+1}=\mathbf{P}_{k}^{*}+T\left[\mathbf{Q}_{k}-\mathbf{S}_{k} \mathbf{R}_{k}^{-1} \mathbf{S}_{k}^{T}-\mathbf{S}_{k} \mathbf{R}_{k}^{-1} \mathbf{H}\left(\hat{\boldsymbol{\theta}}_{k}\right) \mathbf{P}_{k}^{*}+\mathbf{P}_{k}^{*} \mathbf{H}\left(\hat{\boldsymbol{\theta}}_{k}\right) \mathbf{R}_{k}^{-1} \mathbf{S}_{k}^{T}\right] \\
\hat{\boldsymbol{\theta}}_{k+1}=\hat{\boldsymbol{\theta}}_{k}+\left(\mathbf{K}_{k}+T \mathbf{S}_{k} \mathbf{R}_{k}^{-1}\right)\left(\mathbf{y}_{k}-\mathbf{h}\left(\mathbf{y}_{k}, \mathbf{u}_{k}, \hat{\boldsymbol{\theta}}_{k}\right)\right)
\end{gathered}
$$

and the IEKF can propagate its own error covariances, so $\mathbf{Q}_{k}, \mathbf{R}_{k}$ and $\mathbf{S}_{k}$ are now time varying, with the error covariances developed from initial estimates $\mathbf{Q}_{\mathbf{0}}, \mathbf{R}_{\mathbf{0}}$ and $\mathbf{S}_{\mathbf{0}}$ and an ARMA process of the known errors $\boldsymbol{\omega}_{k}$ and $\boldsymbol{v}_{k}$,

with

$$
\begin{gathered}
\mathbf{Q}_{k+1}=(1-\gamma) \mathbf{Q}_{k}+\gamma \lambda^{2} \boldsymbol{\omega}_{k} \boldsymbol{\omega}_{k}^{T} \\
\mathbf{S}_{k+1}=(1-\gamma) \mathbf{S}_{k}+\gamma \lambda \boldsymbol{\omega}_{k} \mathbf{v}_{k}^{T} \\
\mathbf{R}_{k+1}=(1-\gamma) \mathbf{R}_{k}+\gamma \mathbf{v}_{k} \mathbf{v}_{k}^{T} \\
\boldsymbol{\omega}_{k}=\frac{1}{T}\left(\hat{\boldsymbol{\theta}}_{k+1}-\hat{\boldsymbol{\theta}}_{k}\right)
\end{gathered}
$$




$$
\mathbf{v}_{k}=\mathbf{y}_{k+1}-\mathbf{h}\left(\mathbf{y}_{k}, \mathbf{u}_{k}, \hat{\boldsymbol{\theta}}_{k}\right)
$$

Two tuning parameters are now required, $\gamma$ and $\lambda . \gamma$ is a forgetting factor, which determines the influence each individual error vector $\boldsymbol{\omega}_{k}, \boldsymbol{v}_{k}$ has on the error covariances. $\mathbf{Q}_{k}, \mathbf{R}_{k}$ and $\mathbf{S}_{k}$ thus absorb this error information at the rate of a first order lag of time constant $\tau$ where

$$
\gamma=1-e^{-T / \tau}
$$

$\lambda$ performs a similar function to the design covariance $E\left(\boldsymbol{\omega}_{k}^{(\boldsymbol{\theta})} \boldsymbol{\omega}_{k}^{(\boldsymbol{\theta}) T}\right)$, in [6]. Set in the range $0<\lambda<1$, it diminishes the expectation of error in the change in parameters, stabilising the identification. Put simply, the filter causes parameter adaptation which induces (a desirable) non-zero $\dot{\boldsymbol{\theta}}_{k}$. However, these changes are errors according to the zero model of eqn (13), and if their total magnitude is interpreted as error, $\mathbf{Q}_{\mathrm{k}}$ becomes relatively large compared with $\mathbf{R}_{\mathrm{k}}$, which results in an increase in the feedback gain $\mathbf{K}_{\mathrm{k}}$ to provide greater correction to the $\boldsymbol{\theta}$. Subsequent parameter corrections are then larger, and this induces instability. $\lambda$ provides a means of balancing the filter such that changes in $\mathbf{Q}_{\mathrm{k}}$ are, correctly, not interpreted entirely as error.

Initialisation of the filter requires $\mathbf{P}_{\mathbf{0}}, \mathbf{Q}_{\mathbf{0}}, \mathbf{R}_{\mathbf{0}}$ and $\mathbf{S}_{\mathbf{0}}$ to be chosen. A very important primary issue is that the mathematical foundation of the Kalman filter uses trace $\left(\mathbf{P}_{k}\right)$ as the cost. In the IEKF, $\mathbf{P}_{\mathrm{k}}$ is the expectation of parameter error,

$$
\mathbf{P}_{k}=E\left(\left(\hat{\boldsymbol{\theta}}_{k}-\overline{\boldsymbol{\theta}}\right)\left(\hat{\boldsymbol{\theta}}_{k}-\overline{\boldsymbol{\theta}}\right)^{T}\right)
$$

so it is sensible to normalise the parameter set, if equal value is to be placed on each parameter's optimality. This also ensures good conditioning within the filter propagation and makes the filter easier to initialise, since $\mathbf{P}_{\mathbf{0}}$ and $\mathbf{Q}_{\mathbf{0}}$ can be set as multiples of the identity matrix. It turns out that the setting of $\mathbf{P}_{\mathbf{0}}$ is very insensitive and can safely be arbitrarily set. Assuming a nominal 1\% parameter error expectation,

$$
\mathbf{P}_{0}=\hat{\boldsymbol{\theta}}_{0} \hat{\boldsymbol{\theta}}_{0}^{T} \times 10^{-4}
$$

The initial estimate of $\mathbf{R}_{\mathbf{0}}$ can be directly estimated by evaluating a time history for $\mathbf{v}_{k}$ over a suitable section of measurement data $\mathbf{y}$ using the first estimate for the parameter set, $\hat{\boldsymbol{\theta}}_{0}$. From eqn (5), $\mathbf{R}_{\mathbf{0}}$ is then the covariance of the sequence $\boldsymbol{v}_{k}$. It is also reasonable to assume, at least initially, that parameter variations will not correlate with sensor errors, so $\mathbf{S}_{\mathbf{0}}=\mathbf{0}$. Any correlation which does exist will develop naturally within the propagation of $\mathbf{S}_{\mathbf{k}}$ as the filter runs.

$\mathbf{Q}_{\mathbf{0}}$ is less obviously chosen; over long filter run times its influence diminishes, but $\mathbf{Q}_{0}$ does have a more significant role than might be immediately apparent, since its magnitude relative to $\mathbf{R}_{\mathbf{0}}$ determines the initial magnitude of parameter variation, the value of $\boldsymbol{\omega}_{k}$ and hence the propagation of $\mathbf{Q}_{\mathbf{k}}$. Instability is easily avoided through $\lambda<<1$, but $\mathbf{Q}_{\mathbf{0}}$ and $\lambda$ together provide the principal means of controlling the speed of adaptation of the algorithm. As with $\mathbf{P}_{\mathbf{0}}, \mathbf{Q}_{\mathbf{0}}$ can safely be set proportionally to $\hat{\theta}_{0}$, and from eqns (13), (20) and (5), 


$$
\mathbf{Q}_{0}=\lambda^{2} \rho^{2} \hat{\boldsymbol{\theta}}_{0} \hat{\boldsymbol{\theta}}_{0}^{T}
$$

where $\rho$ is the expected proportional rate of change of the parameters - or the desired proportional change of parameters in the first second of running the filter.

The filter can be run in two distinct modes. If relatively high values are chosen for $\lambda$ and $\rho$, with $\tau<1$, the parameters vary in real-time and can track variable conditions such as road surface friction. Alternatively, with low valued $\lambda$ and $\rho$, and with $\tau$ set to the duration of the test data or longer, the parameter change is slow, and the filter can be iteratively passed over the test data to gradually drive the parameters to an identified optimal set.

Performance and stability studies on the setting of all the parameters are given in [5] and [7], so further elaboration is not given here. Settings for $\lambda, \rho$ and $\tau$ to achieve both functions of the filter in the current application, are provided in Sections 4 and 5.

\section{THE IDENTIFIED MODEL}

A 3dof yaw-roll-sideslip model structure is adopted for identification, with fixed roll centres and with the roll axis parallel to the SAE $x$ axis; known model parameters are listed in Table 1 . The principle equations of motion are, for lateral velocity, $v$,

$$
M \dot{v}+M h \dot{p}=\sum_{i=1,4} F_{y i}-M u r
$$

for yaw angular velocity, $r$,

$$
I_{z z} \dot{r}=b \sum_{i=1,2} F_{y i}-c \sum_{i=3,4} F_{y i}
$$

and for roll angular velocity and angle, $p$ and $\phi$,

$$
\begin{aligned}
& \text { Mhi }+I_{x x} \dot{p}=- \text { Mhur }-2 B p+\left(M g h-K_{f}-K_{r}\right) \phi \\
& \dot{\phi}=p
\end{aligned}
$$

where $u$ is the vehicle's longitudinal speed.

The influence of lateral load transfer is included by calculating vertical loads on the tyres using a moment analysis about the roll centre,

$$
\begin{aligned}
& F_{z 1,2}=\frac{c M g}{2(b+c)} \pm \frac{\left(F_{y 1,2} h_{f}-K_{f} \phi-B p\right)}{t_{f}} \\
& F_{z 3,4}=\frac{b M g}{2(b+c)} \pm \frac{\left(F_{y 3,4} h_{r}-K_{r} \phi-B p\right)}{t_{r}}
\end{aligned}
$$

and the tyre slip angles are assumed equal on each axle, with a steering compliance compensation $\left(S_{c}\right.$, scaled in degrees/g) at the front :

$$
\begin{aligned}
& \alpha_{1}=\alpha_{2}=\left(\frac{-v-b r}{u}\right)+\delta-\frac{\pi S_{c} \sum_{i=1,2} F_{y i}}{180 M g} \\
& \alpha_{3}=\alpha_{4}=\left(\frac{r c-v}{u}\right)
\end{aligned}
$$


Each of the four load varying lateral tyre forces $F_{y i}$ are then given by an adaptation of the Pacejka magic formula seen in Milliken and Milliken [8], using slip normalisation such that :

$$
\begin{gathered}
\bar{\alpha}=\frac{G C_{\alpha} \alpha}{P F_{p}} \\
R=\sin \left(C \tan ^{-1}\left(\bar{\alpha} / C-E\left(\bar{\alpha} / C-\tan ^{-1}(\bar{\alpha} / C)\right)\right)\right) \\
F=P F_{p} R
\end{gathered}
$$

This employs four parameters, G,P,C,E which are equivalent to the traditional $B, C, D, E$ quoted in [8]. The reason for the rearrangement is to separate the tyre curve shape parameters, $C, E$ in the new formulae from cornering stiffness gain $(G)$ and peak force $(P)$ coefficients. Both $G$ and $P$ are nondimensional scalar multipliers of the cornering stiffness and peak force functions of vertical load.

Two forms are considered for the tyre model identification. In the basic configuration, G,P,C,E and $S_{c}$ are identified, and vertical load dependence is fixed, based on formulae which have been derived to match previously published (Gordon and Best [9]) variation models :

$$
\begin{aligned}
& C_{\alpha}=7 M g\left(1-e^{-\frac{7 F_{z}}{M g}}\right) \\
& F_{p}=\frac{F_{z}}{1+\left(3 F_{z} / 2 M g\right)^{3}}
\end{aligned}
$$

In the extended model, the five principal tyre parameters are augmented with two further parameters, $\beta_{G}$ and $\beta_{P}$ which allow adaptation of the curvature of the cornering stiffness and peak load functions with vertical load. To achieve this, the original models, eqn (37) are fitted with simple quadratics,

$$
\begin{aligned}
& C_{\alpha}=a_{G} F_{Z}-\frac{\beta_{G} F_{Z}\left(F_{Z}-F_{\text {Zref }}\right)}{1000} \\
& F_{P}=a_{P} F_{Z}-\frac{\beta_{P} F_{Z}\left(F_{Z}-F_{\text {Zref }}\right)}{10000}
\end{aligned}
$$

with parameters $a_{G}=24.9$ and $a_{P}=0.965$, chosen to match the original models exactly at a reference, approximately neutral load for each tyre, $F_{\text {zref }}=4000 \mathrm{~N}$.

In the basic identification, eqns (38) are used, with $\beta_{G}=2.5$ and $\beta_{P}=0.5$ fixed to best reproduce the curvature of eqns (37). In the extended identification $\beta_{G}$ and $\beta_{P}$ are allowed to vary. The original load model, quadratic fit, and adapted quadratic for $C_{\alpha}$ and $F_{P}$ are illustrated within the results section, in Figure 5.

Since the tyre model is load dependent and the load depends directly on lateral tyre force, a lag function is required, which nominally accounts for slip transients in the tyre,

$$
F_{y(k+1)}=\gamma_{y} F_{(k)}+\left(1-\gamma_{y}\right) F_{y(k)}
$$


For ease of coding, the model is executed by explicitly declaring the value of all non-identified parameters, establishing symbolic (Matlab ${ }^{\mathrm{TM}}$ Symbolic Math) descriptions of the state derivatives in terms of the inputs $\mathbf{u}_{k}=\left[u_{k}, \delta_{k}\right]$, and setting

$$
\mathbf{y}=\left[\begin{array}{c}
r \\
v \\
p
\end{array}\right], \quad \mathbf{h}_{k+1}=\mathbf{y}_{k}+T \dot{\mathbf{y}}_{k}, \quad H=\frac{\partial \mathbf{h}}{\partial \theta}
$$

The resulting symbolic expressions are then imported into Matlab code to propagate the IEKF eqns (15) - (24). To avoid excessively long expressions in the Jacobian an intermediate symbolic variable for the lateral tyre forces, $F$ is employed (eqn (36)), and the Jacobian components are calculated using chain rule differentiation;

$$
\frac{\partial h_{m}}{\partial \theta_{n}}=\frac{\partial h_{m}(F)}{\partial \theta_{n}}+\sum_{i} \frac{\partial h_{m}(F)}{\partial F_{i}} \frac{\partial F_{i}}{\partial \theta_{n}}
$$

Table 1 summarises the model's fixed parameter values, which were set where possible using manufacturer's data.

Table 1 Model parameters

\begin{tabular}{|c|l|}
\hline$M$ & Mass $(1840 \mathrm{~kg})$ \\
$I_{z z}$ & Yaw moment of inertia $\left(4140 \mathrm{kgm}^{2}\right)$ \\
$I_{x x}$ & Roll moment of inertia $\left(735 \mathrm{kgm}^{2}\right)$ \\
$b, c$ & CG to front / rear axle distance $(1.69 \mathrm{~m}, 1.34 \mathrm{~m})$ \\
$h$ & CG height above roll axis $(0.41 \mathrm{~m})$ \\
$h_{f / r}$ & Height of front / rear roll centre $(0.1 \mathrm{~m})$ \\
$t_{f / r}$ & Front / rear track $(1.56 \mathrm{~m})$ \\
$K_{f / r}$ & Front / rear roll stiffness $(59 / 36 \mathrm{kNm} / \mathrm{rad})$ \\
$B$ & Roll damping (front / rear equal) $(1225 \mathrm{Nms} / \mathrm{rad})$ \\
$\gamma_{y}$ & Nominal tyre lag factor (as eqn $(25) \mathrm{with} \tau=0.1)$ \\
\hline
\end{tabular}

\section{TYRE MODEL IDENTIFICATION}

The test vehicle is a 2002my X350 3.5l Jaguar XJ8 with identical tyres front and rear. An Oxford Technical Solutions RT3200 combined GPS / inertial measurement system was used to provide all the required data apart from the handwheel steer angle, which was sourced from the vehicle CAN. All data was collected and re-sampled where necessary at $100 \mathrm{~Hz}$ to match the IEKF set with $T=0.01 \mathrm{~s}$.

Identification and validation drives were conducted on a flat, dry proving ground. The identification data comprises a sequence of 10 step steer events in each direction, carried out at cruise controlled constant speed, with each event achieving a steady-state lateral acceleration for a few seconds before returning and again settling to zero steer. Steps with progressively higher magnitude, from $0.2 \mathrm{~g}$ to $0.8 \mathrm{~g}$ were conducted, taking the vehicle slightly beyond the terminal understeer condition (though the combination of rear-wheel drive and cruise control caused nearer neutral / oversteer limit behaviour on occasion, that was suitably controlled by the driver). The sequence was repeated at four fixed speeds, 13, 16, 21 and $24 \mathrm{~m} / \mathrm{s}$, in order to map the tyre behaviour throughout the slip range, but also by including low speeds, through a range of the geometry of the suspension. By including the full slip and geometry operating ranges of the tyre, the 
resulting system identification can provide a suitably averaged tyre model. To confirm this and to test the model on a different type of test input, validation data comprised a 'free drive' around the proving ground with low frequency speed variations and a combination of low and high frequency steer manoeuvres.

The lateral velocity - which is clearly critical to successful identification and is also the most difficult signal to accurately track - changes its sign with respect to steer angle as the vehicle passes from lower to higher speeds; the two signals are positively correlated below, and negatively correlated above $18 \mathrm{~m} / \mathrm{s}$ in this test vehicle. This is an expected result which is seen clearly in the vehicle data, and which can be readily verified with the model by considering slip and yaw moment balance at the rear axle. Identification with data collected at around $18 \mathrm{~m} / \mathrm{s}$ is therefore omitted to avoid conditioning problems associated with very low amplitude lateral velocity signals around this speed.

The IEKF adaptation speed parameters are set at $\tau=350, \lambda=0.01$ and $\rho=0.1$, to encourage slow, repeatable model parameter identification, and the 350 second vehicle test data is applied iteratively, with Kalman gain and error matrices continuously adapting across iteration boundaries. The initial and final model parameters for both basic and extended tyre models are given in Table 2, and Figures 1-5 illustrate the convergence, performance and tyre models which are generated.

Table 2 Initial and converged parameter values (400 iterations)

\begin{tabular}{|l|c|c|c|c|c|c|c|}
\hline & $P$ & $G$ & $C$ & $E$ & $S_{c}$ & $\beta_{G}$ & $\beta_{P}$ \\
\hline $\boldsymbol{\theta}_{0}$ Initial condns & 1.10 & 1.00 & 1.40 & -0.20 & 2.00 & 2.50 & 0.50 \\
\hline $\boldsymbol{\theta}_{\text {opt }}$ Basic model & 1.02 & 1.28 & 1.24 & -1.57 & 4.38 & $(2.50)$ & $(0.50)$ \\
\hline $\boldsymbol{\theta}_{\text {opt }}$ Extended model & 1.26 & 1.09 & 1.25 & -0.20 & 3.93 & -1.75 & 1.09 \\
\hline
\end{tabular}

First it is important to recognise that the initial parameters for the tyre and (more critically) for the model (Table 1) have not been set arbitrarily. They are chosen to match the test vehicle as closely as possible, and in addition, the centre of gravity geometry variables ( $b$ and $h$ ) have been optimised by a prior IEKF experiment, described in [10]. It would be trivial to present a poorly matched initial parameter set and hence achieve apparently remarkable performance improvements here; instead the objective is to see the marginal benefits of the optimised tyre model.

\section{[Figure 1]}

Figure 1 shows cost and parameter convergence, with a subset of parameters chosen to illustrate typical behaviour. A single iteration takes approximately 1 minute on the mid-range PC used here, so we can see from the figure that an effective, low cost tyre model is available within around 10 minutes, whereas several hours are needed to find the definitive identification result. If used within a real-time vehicle controller, the slow adaptation would not present a problem provided environmental parameters are adapted more rapidly - as we will see in Section 5.

There is a distinct difference between convergence rates in the parameters, with the most cost-sensitive, such as $P$ converging quickly. Other parameter combinations, such as $C$ (not shown) and $E$ vary slowly but achieve very little additional cost reduction seen in trace $(R)$. The $\beta$ parameters also illustrate this, with $\beta_{P}$ converging much more rapidly than $\beta_{G}$. Separate, six parameter extended model optimisations have been conducted with $\beta_{G}$ fixed, and these confirm that most of the cost benefit of including the $\beta$ terms arises from the identification of the peak force model.

The extension of the model does bring about a considerable variation in the final values of common parameters though - eg in $E$ and in the alternated choice of higher $P$ 
or $G$ (table 1); this illustrates their known interdependence. Note also that Figure 1 shows $E$ varying away from its $\theta_{0}$ setting in the first few iterations, before converging to its original value, so this parameter has not been neglected in the identification.

With the possible exception of $S_{c}$, all of the parameters converge to values within the expected range. The initial setting, $S_{c 0}=2$ fits with an assumption, supported by the manufacturer, that the front suspension might reasonably see compliance in the region of $1 \%$ g (see also eqn (33)). The higher converged setting is not outside reasonable bounds of variability, but we should also add the caveat that this value corrects any calibration inaccuracies in the measured steer angle, and more significantly, will be affected by body torsional compliance as well as the assumption that front left and right slip and steer angles are equal. Critically, the inclusion of the compliance term is extremely important to the high levels of accuracy in state predictions seen in this paper; the term is not included in most published handling models, yet it plays an important role in differentiating front / rear tyre force, suspension and vehicle behaviour here.

[Figures 2 \& 3]

Table 3 Open-loop Model performance \%, RMS(error) / RMS(source data)

\begin{tabular}{|l|c|c|c|c|}
\hline Identification data & yaw rate, $r$ & lat. vel, $v$ & roll rate, $p$ & lat. accel, $a_{y}$ \\
\hline $\boldsymbol{\theta}_{0}$ Initial conditions & 16.3 & 148.0 & 72.0 & 25.1 \\
\hline $\boldsymbol{\theta}_{\text {opt }}$ Basic model & 5.2 & 41.4 & 61.1 & 8.5 \\
\hline $\boldsymbol{\theta}_{\text {opt }}$ Extended model & 5.4 & 35.9 & 62.5 & 8.2 \\
\hline & & & & \\
\hline Validation data & yaw rate, $r$ & lat. vel, $v$ & roll rate, $p$ & lat. accel, $a_{y}$ \\
\hline $\boldsymbol{\theta}_{0}$ Initial conditions & 22.7 & 116.9 & 71.1 & 24.3 \\
\hline $\boldsymbol{\theta}_{\text {opt }}$ Basic model & 8.7 & 70.7 & 64.0 & 11.2 \\
\hline $\boldsymbol{\theta}_{\text {opt }}$ Extended model & 8.8 & 70.2 & 67.3 & 10.7 \\
\hline
\end{tabular}

Figures 2 and 3 show a representative section of 'open-loop' model performance on the identification and validation data sets respectively, and Table 3 quantifies the RMS errors between source and simulated data. Note that these are a worst case illustration of the value of the model, since the variables are simulated in isolation from the source data, based on the measured inputs $\mathbf{u}_{k}=\left[u_{k}, \delta_{k}\right]$ alone. For both identified models, performance is improved on all of the model states, but the improvement is clearest in lateral velocity.

The identified tyre seems equally effective in basic and extended forms, with just a small further improvement noticeable for the extended tyre variant for lateral velocity. It is however, relatively easy to tune parameters for the yaw-roll-sideslip model to achieve excellent yaw rate and lateral acceleration tracking; it is the often very sensitive lateral velocity measure which is critical to full state feedback chassis control systems, so the improved prediction from the tyre model here might provide significant value.

Normalised lateral force vs slip curves for both identified tyre models are given in Figure 4, and the load dependency models are shown in Figure 5. In Figure 4, given the measurement of yaw and lateral accelerations along with all of the constituent variables required to formulate tyre slip angles, it is possible to construct a scatter plot of the normalised tyre behaviour directly from the measurements. This is not independent of an assumed tyre and vehicle model, but it does provide a useful measure of the consistency of the tyre behaviour and hence the suitability of a single tyre model for all wheelstations.

To generate the scatter plot, cumulative axle force is deduced from measured lateral 
and yaw accelerations $a_{y}$ and $\dot{r}$ :

$$
\left[\begin{array}{c}
F_{y f} \\
F_{y r}
\end{array}\right]=\left[\begin{array}{cc}
1 & 1 \\
b & -C
\end{array}\right]^{-1}\left[\begin{array}{c}
M a_{y} \\
I_{z z} \dot{r}
\end{array}\right]
$$

An estimate of vehicle roll angle,

$$
\hat{\phi}=\frac{-\left(F_{y f}+F_{y r}\right) h}{K_{f}+K_{r}+M g h}
$$

is then applied to eqn (32) to estimate the tyre vertical loads. (Measured roll angle suffers from low frequency errors since it is an absolute measure and small road camber angles are not insignificant and unknown.)

Measured $\delta, u, v$ and $r$ are then applied to eqns (33) - (36) and (38), using the identified tyre parameters, to find $\bar{\alpha}$ and F; $F$ is used to attribute an appropriate left / right split to the 'measured' data $F_{y f}$ and $F_{y r}$, and hence find an equivalent 'measured' $R$ by inversion of eqn (36) :

$$
R_{1}=\left(\frac{F_{1}}{F_{1}+F_{2}}\right) \frac{F_{y f}}{P F_{p}}, R_{2}=\left(\frac{F_{2}}{F_{1}+F_{2}}\right) \frac{F_{y f}}{P F_{p}} \text { etc }
$$

The four measurement based $R_{i}$ and $\bar{\alpha}_{i}$, plotted as the scatter in Figure 4 therefore show how the measurements on the identification drive emulate eqn (35). Alterations to parameter choices will obviously influence both the model curve and scatter data in the plot, but it is clear both that the expected shape exists, and that the four corners provide consistent behaviour, validating the single tyre assumption. Note however that direct optimisation of parameters from this plot cannot be achieved, since (eg) $P \rightarrow \infty$ or $G \rightarrow 0$ tend to a zero slip null solution.

It is interesting that although peak forces, $P$ are consistent, Figure 4 shows a mismatch in the cornering stiffness, $G$ seen in the model and scatter data; the model shows higher stiffness. Given that both of these data sources are generated using the parameters $G$ and $P$, we might expect a better match. Note however that the tyre model is optimised in combination with the vehicle model in order to achieve minimum error in the vehicle states; this is not a tyre force optimisation in isolation, and the objective function is not lateral force and slip. The higher $G$ is compensated in the model by suspension compliance $S_{c}$, and it also counteracts the vehicle model simplicity and its fixed parameter choices to provide the excellent state tracking of Figures 2 and 3. Figure 4 thus provides a useful resource for future tuning and / or extension of the vehicle model structure, to achieve a more accurate tyre model independently from the vehicle and its suspension.

Extension of the identification has the effect of spreading the range of normalised slip values - an effect caused principally by the alternative to the $F_{y p}$ model. In Figure 5 (a) the cornering stiffness model has become nearly linear - in effect cancelling out the influence of this parameter on the axle's aggregated tyre force. Conversely the peak force model is identified with the expected shape, but with more exaggerated saturation at high vertical load. Noting the marginal performance improvements in Table 3 and the fact that the extended cornering stiffness model now varies considerably from expectations, we should exercise some caution about the applicability of the extended model. It could be argued that the improvements are due more to the increased parameter freedom than improved accuracy of the tyre model itself. 


\section{RAPID ADAPTATION TO ROAD SURFACE FRICTION}

Having successfully identified the tyre model in consistent, dry proving ground conditions, it is convenient that the IEKF also has the ability to adapt rapidly, so can also be used to track road surface friction in real-time. With most of its parameters fixed, either of the identified tyre models can be used, with either $P$ or $G$ rapidly adapting.

Variation of $P$ will vertically scale the tyre force curve, whereas $G$ will alter the linear range, cornering stiffness behaviour. Tests have been conducted predominantly in the low lateral acceleration range, and consequently $G$ variation is the most successful option. For prior warning of low surface friction, estimators which operate at low lateral accelerations are preferable in any case.

Conversion of the IEKF is straightforward; $\boldsymbol{\theta}=G$, and the three adaptation parameters are reset to $\tau=0.01, \lambda=0.1$ and $\rho=0.003$. Particularly critical settings here are $\tau$ and $\lambda$; with $\tau$ set so short, the error covariance matrices will not settle, and with $\lambda$ ten times higher than for the identification experiments, the parameter will vary rapidly.

Two test scenarios are considered; the first is a drive on a wet handling proving ground circuit equipped with a large circular section with three tracks on separate radii, each with a different road surface, with friction ranging from $\mu \approx 0.3$ to $\mu \approx 0.75$. Approach sections of track were kept dry, giving $\mu \approx 1$. This track was driven without cruise control, at speeds ranging from 10 to $20 \mathrm{~m} / \mathrm{s}$ and with accelerations up to $5 \mathrm{~m} / \mathrm{s}^{2}$. GPS tracking allowed subsequent reconstruction of the expected friction level at each part of the time history.

The second scenario is a control case, whereby a section of normal driving on a dry, twisting country road was analysed; this driving saw a similar range of vehicle speed and lateral accelerations.

\section{[Figures 6 and 7]}

The results are presented in Figures 6 and 7 respectively, with plots (a) showing the real-time estimation of road friction. The variation in $G$ tracks $\mu$ reasonably closely on the wet handling circuit, and remains consistently very close to the expected $\mu=1$ on the control road. One disadvantage, though inevitable for any handling dynamics based estimator, is that the IEKF responds only to transients, so correction may be delayed when there is zero or near zero steer input. This is true for the intervals 95-105 seconds and 220-230 seconds on Figure 6(a) where we can see delays in the change in $\mu$. However, when dynamic activity coincides with $\mu$ changes - eg at 125 seconds, the $\mu$ estimation changes quickly - here within 5 seconds. Figure 6(b) shows a 100 second section of the test over which friction variations occur, and we see a clear improvement in lateral velocity tracking in the adaptive case. In Figure 7(b) and (c) the tracking difference is not detectable due to the small $\mu$ variation, and these plots also serve to illustrate the difference between the model capacity in the different states; the performance in $v$ is good, but as with all the model variants in the paper, tracking of $a_{y}$ is exceptional.

\section{CONCLUSION}


The results confirm that it is possible to identify a single tyre model which operates consistently for all four wheelstations, provided some compensation is made for the compliance variation expected from front to rear differences in suspension design.

The tyre model is identified within the context of a simple vehicle handling model, and the state prediction performance of this model improves for all states when the tyre parameters are optimised. Significantly, the lateral velocity state prediction is most improved, making the combined model particularly applicable for control design applications. Expansion of the tyre model to include variation of load dependence parameters provides a small further improvement in lateral velocity prediction performance, though with some degradation to expected cornering stiffness vs load behaviour

In general, the algorithms employed here can be used to generate vehicle handling models for control applications. They can also be used to identify the holistic behaviour of a given tyre choice on vehicle response, and to see the interaction effects of the tyre with the suspension.

Identified tyre models can further be complemented by the IEKF operating in realtime to rapidly adapt the cornering stiffness term, and hence almost instantaneously compensate for changes in road friction.

In summary, in both off-line and real-time applications, the IEKF represents a valuable tool in the identification and adaptation of vehicle handling models, and although model complexity must remain reasonably low, it also provides an excellent means of identifying tyre and road friction behaviour directly from vehicle test data.

\section{REFERENCES}

[1] Radt, H.S. and Pacejka, H.B., "Analysis of the Steady-State Turning Behavior of an Automobile", Proceedings of the Symposium on Control of Vehicles, Instn Mech. Engrs, London, 1963.

[2] Pacejka, H.B. and Besselink, I.J.M., (1996) "Magic Formula Tyre Model with Transient Properties”, Vehicle System Dynamics, 27:1, 234-249.

[3] Canudas-de-Wit, C. et al., (2003) “A New Nonlinear Observer for Tire/Road Distributed Contact Friction”, Proceedings of the IEEE Conference on Decision and Control, Vol 3, pp 2246 - 2251.

[4] Bolzern, P., Cheli., F., Falciola, G. and Resta, F., "Estimation of the Non-Linear Suspension Tyre Cornering Forces from Experimental Road Test Data”, Vehicle System Dynamics, 1999, vol 31 no 1, pp 23-34.

[5] Best, M.C., Newton, A.P. and Tuplin, S, “The identifying extended Kalman filter: parametric system identification of a vehicle handling model”, Proc. Instn Mech. Engrs, Part K: J. Multi-body Dynamics, 2007, 221(O1), 87-98.

[6] Best M.C., Gordon T.J. and Dixon P.J., "An Extended Adaptive Kalman Filter for Real-time State Estimation of Vehicle Handling Dynamics”, Vehicle System Dynamics, 2000, vol 34, no 1, pp 57-75.

[7] Best, M.C., "Parametric identification of vehicle handling using an extended Kalman filter”, International Journal of Vehicle Autonomous Systems, Vol 5, No 3 / 4, 2007, pp 256 - 273.

[8] Milliken W.F. and Milliken D.L., "Race Car Vehicle Dynamics”, SAE 
International, 1995.

[9] Gordon, T.J., and Best, M.C., "On the Synthesis of Driver Inputs for the Simulation of Closed-loop Handling Manoeuvres”, International Journal of Vehicle Design, 2006, Vol. 10, No. 1/2/3 pp 52-76.

[10] Best, M.C. and Newton, A.P., "Vehicle Tyre and Handling Model Identification using an Extended Kalman Filter" proceedings from the $9^{\text {th }}$ International Symposium on Advanced Vehicle Control (AVEC), Kobe, Japan, October 2008. 


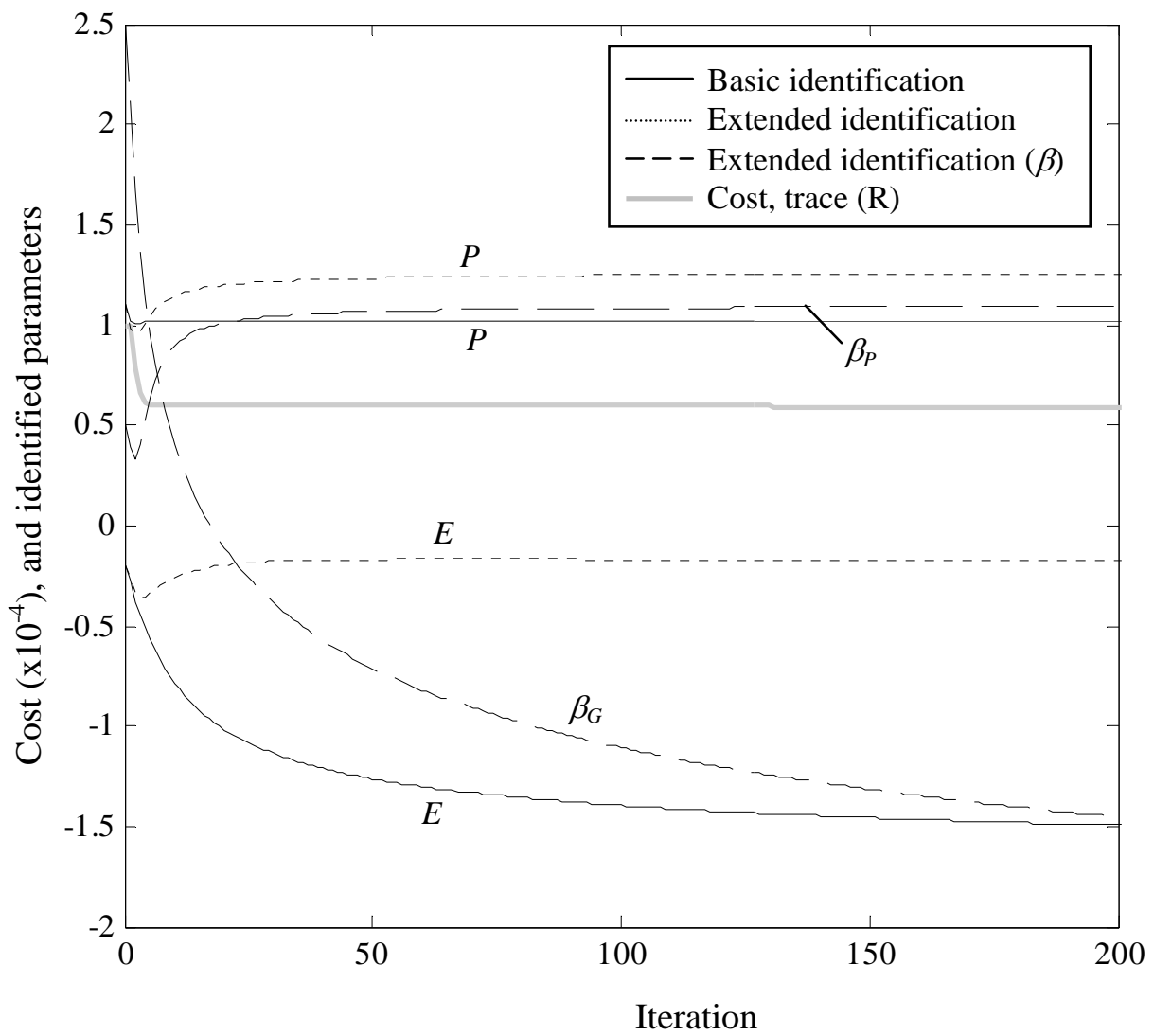

Figure 1 : Cost and parameter convergence 


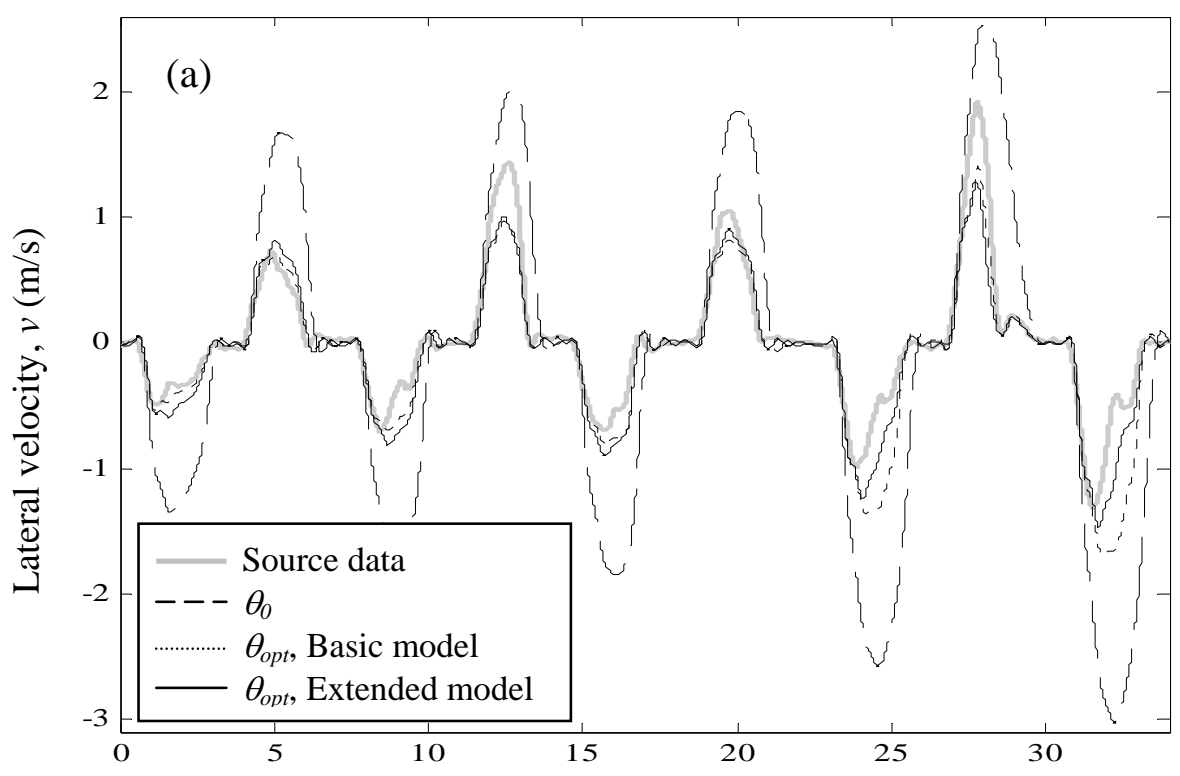

(b)

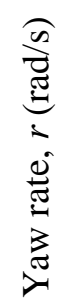

Time (s)

Figure 2 : Open-loop state performance on identification data with forward speed $u=24 \mathrm{~m} / \mathrm{s}$

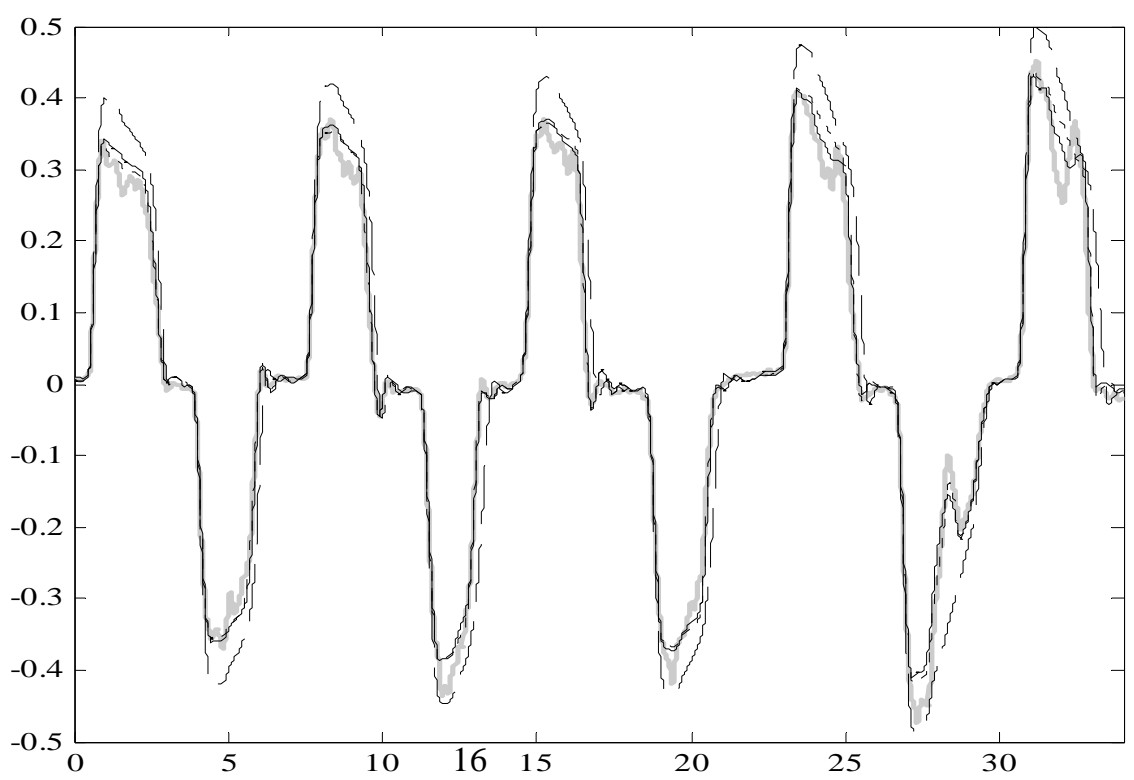



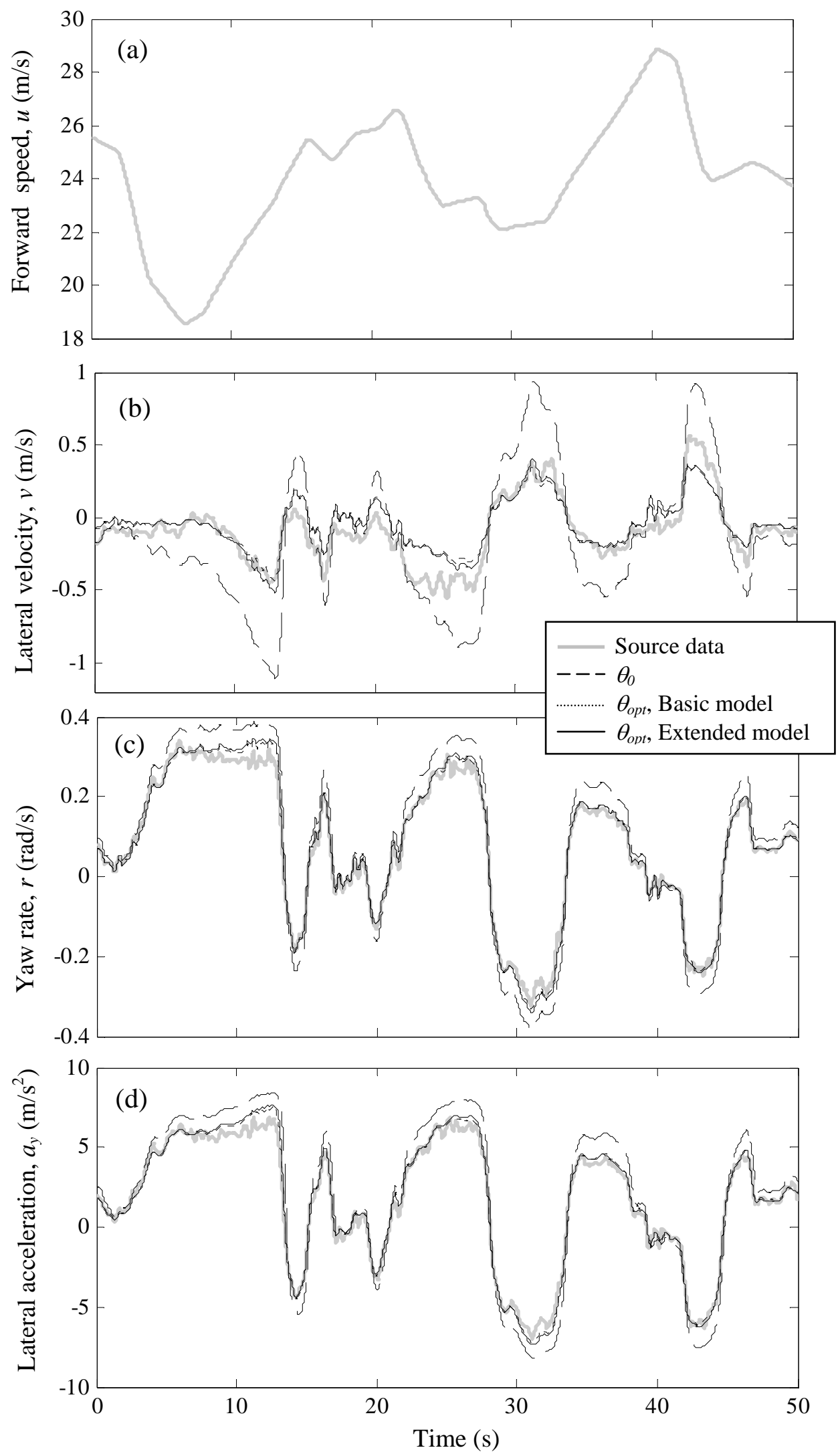

Figure 3 : Open-loop state performance on validation data 

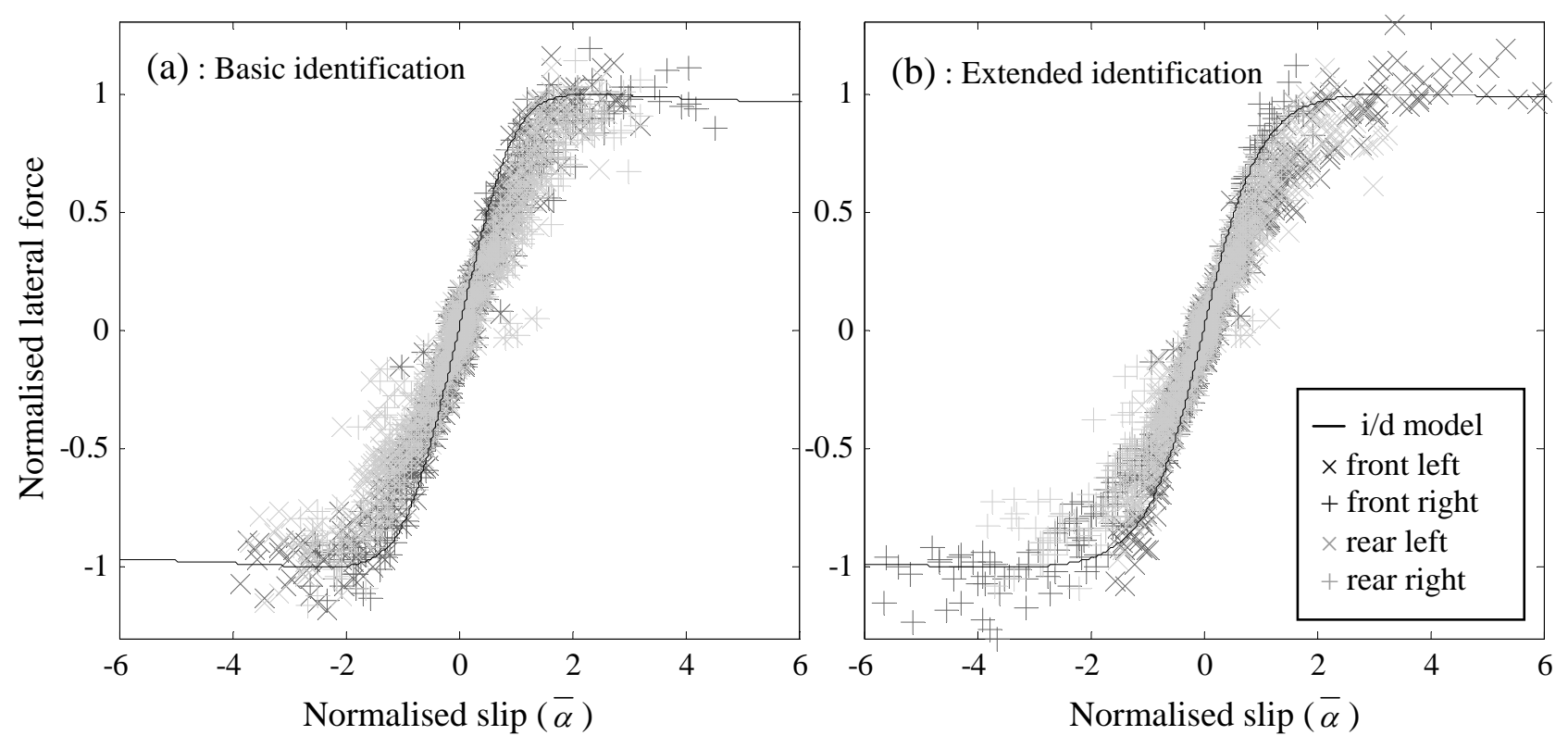

Figure 4 : Normalised tyre models illustrated against approximating tyre data 

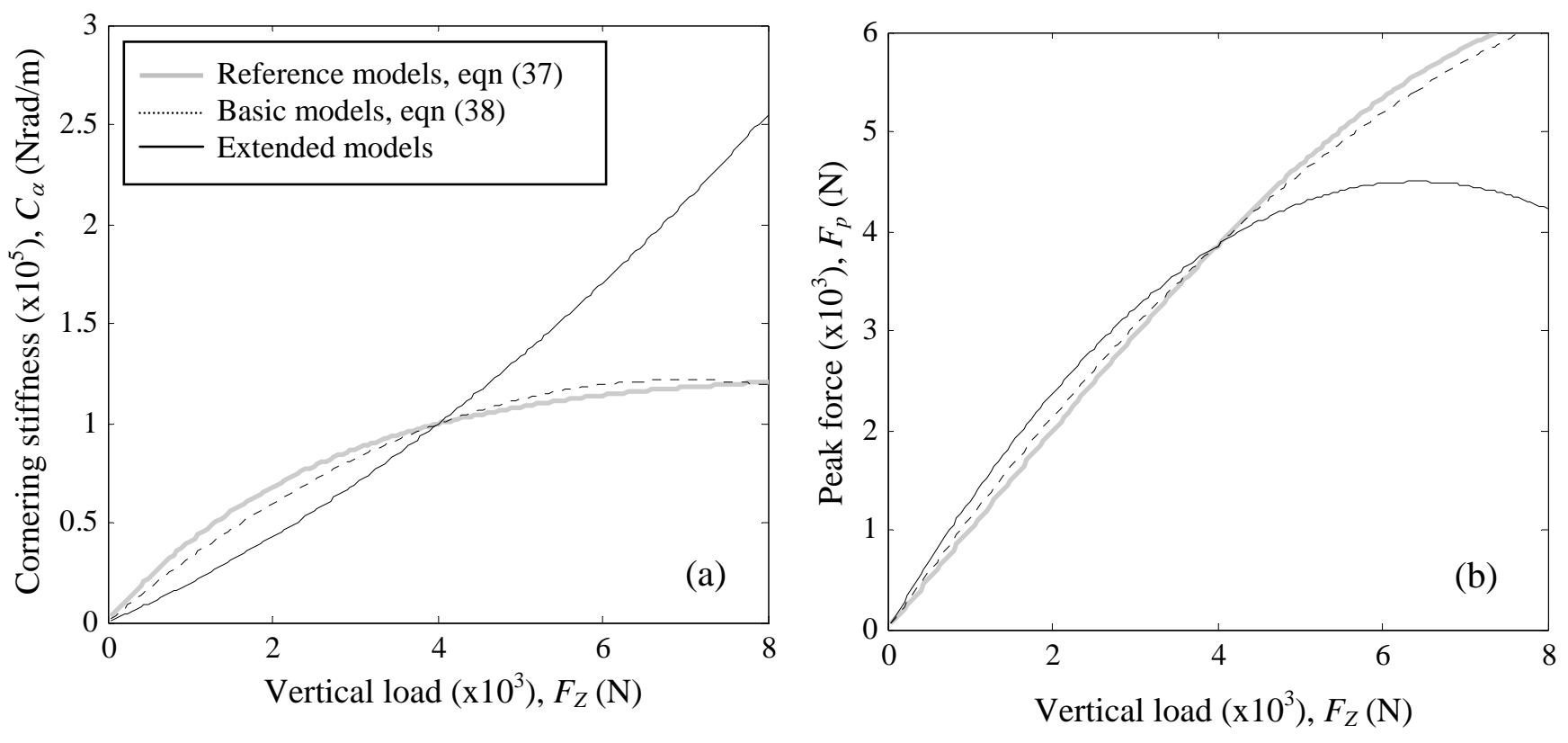

Figure 5 : Tyre load dependence models 

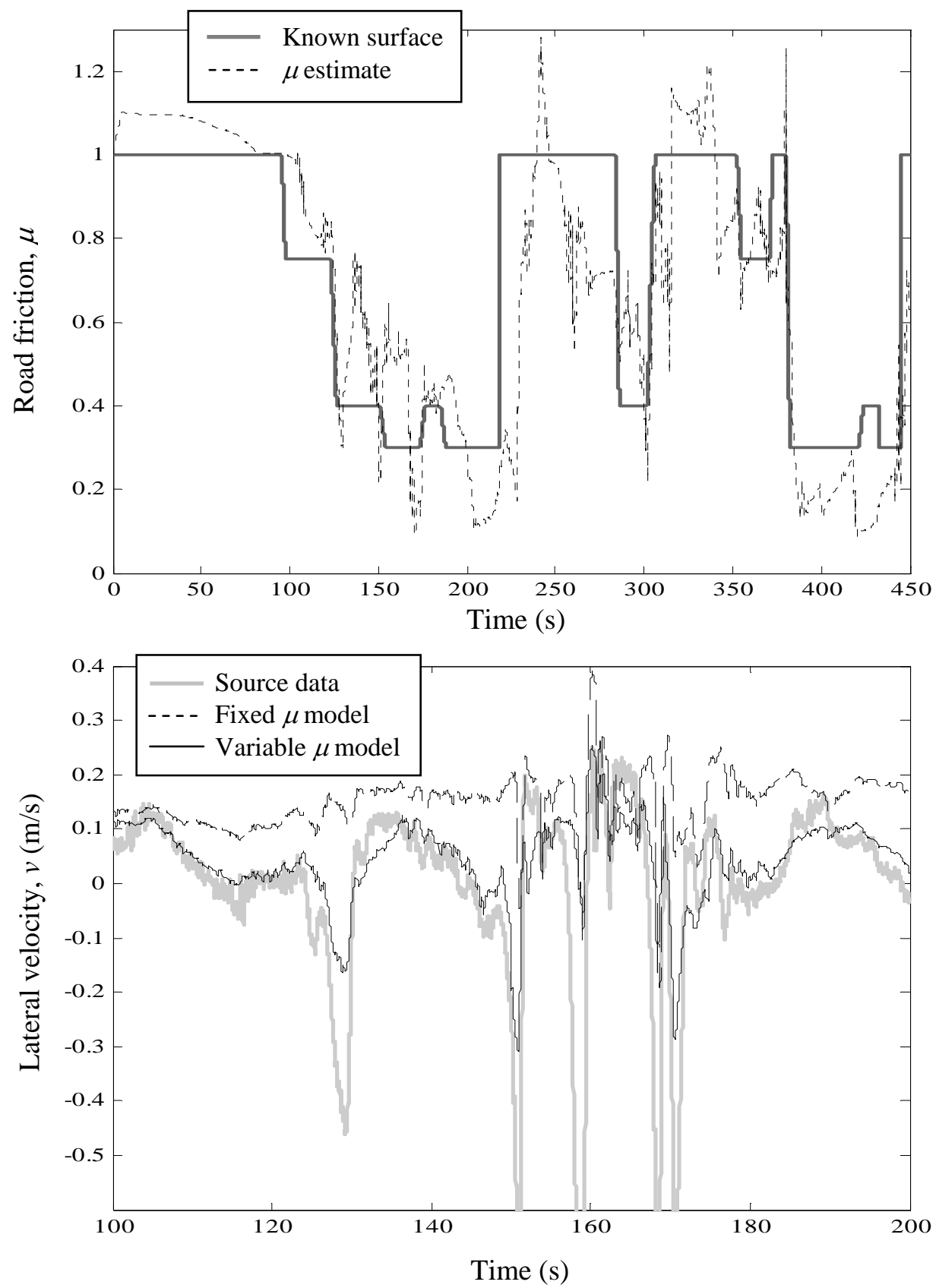

Figure 6 : Real-time road friction estimation and state tracking; wet handling test track 

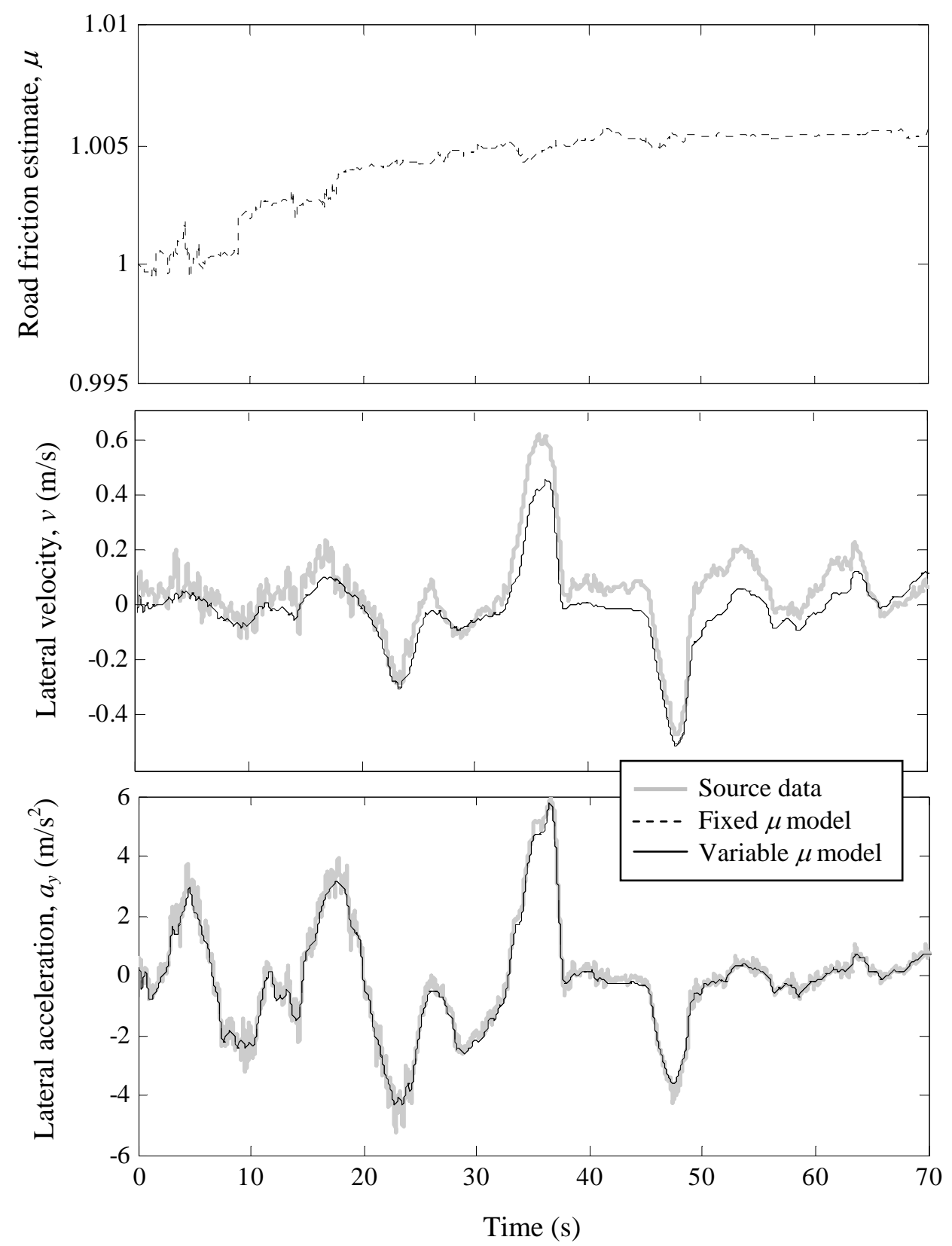

Figure 7 : Real-time road friction estimation and state tracking; dry tarmac road 